\title{
New information on morphology and molecular data of camallanid nematodes parasitising Xenopus laevis (Anura: Pipidae) in South Africa
}

\author{
Roman Svitin ${ }^{1,2}$, Anneke L. Schoeman ${ }^{1,3}$ and Louis H. du Preez ${ }^{1,4}$ \\ ${ }^{1}$ African Amphibian Conservation Research Group, Unit for Environmental Sciences and Management, North-West University, \\ Potchefstroom, South Africa; \\ ${ }^{2}$ Department of Invertebrate Fauna and Systematics, I. I. Schmalhausen Institute of Zoology, Kyiv, Ukraine; \\ ${ }^{3}$ Centre of Excellence for Invasion Biology, Unit for Environmental Science and Management, North-West University, \\ Potchefstroom, South Africa; \\ ${ }^{4}$ South African Institute for Aquatic Biodiversity, Grahamstown, South Africa
}

\begin{abstract}
Three species of nematodes from the Camallanidae that are known to infect Xenopus laevis Daudin (Anura: Pipidae) were collected from several localities across South Africa. New data on morphology, partial 28S and coxl genes, infection levels and distribution are presented herein. The most common species, Batrachocamallanus slomei Southwell et Kirshner, 1937, from the stomach and less often oesophagus, was found in eight localities. Camallanus kaapstaadi Southwell et Kirshner, 1937, also from the oesophagus, was found in two localities and C. xenopodis Jackson et Tinsley, 1995, from the intestine, at a single locality. New localities for both C. kaapstaadi and C. xenopodis provide a geographical range extension. Males of C. xenopodis are described for the first time herein. The existence of a left spicule in the males of both the species of Camallanus Railliet and Henry, 1915 is confirmed and measurements are provided. Although C. xenopodis is distinguished from C. mazabukae Kung, 1948 in the present study, we suggest greater sampling effort in other African amphibians to confirm the species status of the latter taxon. Finally, the new molecular data showed distant relationships between collected species of Camallanus and species parasitising fish and freshwater turtles.
\end{abstract}

Keywords: African clawed frog, parasites, Nematoda, Camallanidae, Camallanus, C. kaapstaadi, C. xenopodis, Batrachocamallanus, B. slomei, Procamallanus

Over 25 parasite genera from seven invertebrate groups have been reported to be associated with the African clawed frog Xenopus laevis Daudin (Anura: Pipidae) in its native southern African range (Tinsley 1996). Some of these parasites have followed their invasive hosts out of Africa to North America, as well as to Europe (Lafferty and Page 1997, Kuperman et al. 2004). One group of parasites that, to date, has not been reported from feral populations of $X$. laevis is the nematodes of the family Camallanidae that primarily parasitise marine and freshwater fish and less often amphibians, turtles and snakes (Stromberg and Crites 1974).

In clawed frogs across the southern African subcontinent, representatives of camallanids are widespread - Kenya, Nigeria, Rwanda, Uganda and Zimbabwe (Thurston 1970, Avery 1971, Tinsley et al. 1979). Specifically, three species of camallanids are associated with $X$. laevis in South Africa: Camallanus kaapstaadi Southwell et Kirshner, 1937, C. xenopodis Jackson et Tinsley, 1995 and $B a-$ trachocamallanus slomei (Southwell et Kirshner, 1937).
Camallanus kaapstaadi was described from X. laevis from Cape Town (South Africa) (Southwell and Kirshner 1937). Subsequently, it was identified from clawed frogs (X. laevis and other Xenopus spp.) from Cameroon, Ghana, Kenya, Nigeria, Rwanda, northern and south-eastern parts of South Africa (previously known as Transvaal and Transkei), Sudan, Tanzania, Togo, Uganda and Zimbabwe (Avery 1971, Jackson and Tinsley 1995a).

Furthermore, the junior synonym of $C$. kaapstaadi, C. johni Yeh, 1960, was described from Tanzania (Yeh 1960) and later also reported from Zimbabwe, Uganda and Nigeria under this name (Thurston 1970). In the meantime, Tinsley et al. (1979) recorded the presence of a species of Camallanus Railliet and Henry, 1915 in X. wittei Tinsley, Kobel et Fischberg from central Africa, which was later identified as C. kaapstaadi (Jackson and Tinsley 1995a). Therefore, C. kaapstaadi can be regarded as a widespread parasite of clawed frogs across Africa.

The second camallanid from $X$. laevis, C. xenopodis, was described based only on six female specimens from 
the Cape (South Africa) and one female specimen which was recovered in Kenya from $X$. borealis Parker (Jackson and Tinsley 1995a). Neither of these species has been recorded since.

The third species, B. slomei, was also described as Procamallanus slomei Southwell and Kirschner, 1937 from $X$. laevis from the Cape (South Africa) (Southwell and Kirshner 1937) and was formally recorded for the first time since its original discription as B. slomei from clawed frog hosts in South Africa and Zaire (Jackson and Tinsley 1995b). Jackson and Tinsley (1995b) also synonymised Procamallanus brevis Kung, 1948 from X. laevis in South Africa (Kung 1948) with B. slomei and reidentified a Spirocamallanus xenopodis Southwell and Kirschner, 1937 in a clawed frog from Kenya (Thurston 1970) as belonging to the same species.

The redescription of $B$. slomei as a member of the genus Batrachocamallanus Jackson et Tinsley, 1995 is not without controversy (Jackson and Tinsley 1995b). The genus Batrachocamallanus was erected specifically for the four species of Procamallanus Baylis, 1923 parasitising African amphibians, based upon the large number of mucrons (more than five) on the female tail, relatively smaller body size and the almost identical cephalic morphology, male caudal structures and female reproductive system that differentiate the amphibian parasites from all other species of Procamallanus (see Jackson and Tinsley 1995b).

Moravec et al. (2006) considered differences in female mucron number and affinity to an amphibian host as not sufficient generic characters and advocated for the reduction of Batrachocamallanus to a junior synonym of Procamallanus. This decision was based on the description of Procamallanus pacificus Moravec, Justine, Würtz, Taraschewski et Sasal, 2006 from Pacific eels (Anguilla sp.) (Actinopterygii: Anguillidae) bearing four to nine mucrons on the female tail (Moravec et al. 2006) and the discovery of B. siluranae Jackson et Tinsley, 1995, previously described from Xenopus tropicalis Gray (Anura: Pipidae), from the polypteroid fish Erpetoichthys calabaricus Smith (Actynopterigii: Polypteridae) (Řehulková et al. 2005).

However, only one immature female specimen of $B$. siluranae was recovered from the polypteroid fish, which was a specimen imported for the pet trade, leading the authors to believe this to be an accidental infection ( ová et al. 2005). Moreover, the small processes on the tail of female $P$. pacificus are $6 \mu \mathrm{m}$ to $9 \mu \mathrm{m}$ long whereas the mucrons of Batrachocamallanus spp. vary from $10 \mu \mathrm{m}$ to $15 \mu \mathrm{m}$ (Jackson and Tinsley 1995b, Moravec et al. 2006). Therefore, the mucrons of $P$. pacificus might not represent the same structures as in Batrachocamallanus and probably cannot be used for suppression of the genus. Taken as a whole, these factors cast doubt on the validity of the suppression of Batrachocamallanus, leading us to assign our specimens to B. slomei.

It is clear that information on the camallanids from $X$. laevis are in need of augmentation, especially on the molecular front. The present studies of three species of camallanids collected from $X$. laevis in different regions of South Africa add information on the infection level, locali- ties, morphology and molecular data of the 28S rRNA and coxl genes.

\section{MATERIALS AND METHODS}

In total, 97 Xenopus laevis were collected in chicken liver baited funnel traps from eight localities across the northern regions of South Africa during November of 2016 and March to May of 2017 and from three localities in the south-western regions during June and July of 2017 (Table 1).

The hosts were anaesthetised in 6\% ethyl-3-aminobenzoate methanesulfonate (MS222) (Sigma-Aldrich Co., St. Louis, Missouri, USA) and subsequently euthanised through severing the spine and destroying the brain, according to internationally accepted standard operating procedures. During the total dissection, the alimentary canal was removed and opened in $0.6 \%$ amphibian saline. After removal from the oesophagus, stomach and intestine, nematodes were washed in saline, fixed in $70 \%$ hot ethanol and subsequently stored in $70 \%$ ethanol.

Prior to microscopic examination, the nematodes were cleared in lactophenol. The morphology of the nematodes was studied and photomicrographs were taken using a Nikon E800 and Nikon ECLIPSE Ni compound microscopes. Apical and transverse sections were prepared manually.

In total, 601 specimens of B. slomei, 39 of C. kaapstaadi and 53 of $C$. xenopodis were studied of which 50 (20 males and 30 females), 18 (nine males and nine females) and 28 (four males and 24 females), respectively, were measured. All measurements in the text are given in micrometres, unless otherwise indicated and presented as ranges followed by mean values in parentheses. The representative sample ( 30 specimens) expressed as mean with standard deviation (SD).

For the molecular studies, the middle fragments of males were used, while taxonomically important anterior and posterior parts were reserved for the morphological identification of species. DNA was extracted using the KAPA Express Extraction Kit DKAPKK7103 (Sigma-Aldrich Co.). Cox1 amplicons were obtained using the primer pair 'LCO1490' (5'-GGTCAACAAATCATAAAGATATTGG-3') and 'HCO2198' (5'-TAAACTTCAGGGTGACCAAAAAATCA-3'). The thermocycling profile was: 3 min denaturation at $94^{\circ} \mathrm{C}, 10$ cycles of $94^{\circ} \mathrm{C}$ for $30 \mathrm{~s}, 45^{\circ} \mathrm{C}$ for $30 \mathrm{~s}, 72^{\circ} \mathrm{C}$ for $60 \mathrm{~s}$ and 40 cycles of $94^{\circ} \mathrm{C}$ for $30 \mathrm{~s}, 51^{\circ} \mathrm{C}$ for $60 \mathrm{~s}, 72^{\circ} \mathrm{C}$ for $60 \mathrm{~s}$ for amplification and $72^{\circ} \mathrm{C}$ for $10 \mathrm{~min}$ for extension.

The 28S amplicons were amplified using forward primer 'LSU5' (5'-TAGGTCGACCCGCTGAAYTTAAGCA-3') and reverse primer 'n900r' (5'-GGTTCGATTAGTCTTTCGCC-3'). The thermocycling profile was as follows: 5 min denaturation at $95^{\circ} \mathrm{C} ; 40$ cycles of $30 \mathrm{~s}$ at $95^{\circ} \mathrm{C}, 30 \mathrm{~s}$ at $55^{\circ} \mathrm{C}, 2 \mathrm{~min}$ at $72^{\circ} \mathrm{C}$ for amplification; and a final $7 \mathrm{~min}$ extension at $72^{\circ} \mathrm{C}$. Sequences were obtained using BigDye ${ }^{\circledR}$ Terminator v3.1 Cycle Sequencing on an $\mathrm{ABI} 3500 \mathrm{XL}$ sequencer. DNA products were sequenced in both directions using the pairs of PCR primers; for the nuclear genes the following additional primers were used: internal primers, '300F' (5'-CAAGTACCGTGAGGGAAAGTTG-3') and 'ECD2' (5'-CTTGGTCCGTGTTTCAAGACGGG-3'). Contiguous sequences were assembled, edited using Geneious 9.0 software and submitted to GenBank.

For the phylogenetic analysis, Bayesian inference in the $\mathrm{Mr}$ Bayes program (V. 3.2.2) was used. Prior to analysis, sequences 
Table 1. Geographic origin, habitat and level of infection of three nematodes of 97 Xenopus laevis Daudin from 11 localities in South Africa. Mean intensity of infection is given with median intensity in square brackets and minimum and maximum values in parentheses. Fields with ' $n / a$ ' refer to absent infection level values due to absence of nematodes in the digestive tract.

\begin{tabular}{|c|c|c|c|c|c|c|c|}
\hline Nematode & Localities & Coordinates & Habitat & $\begin{array}{l}\text { Number } \\
\text { of hosts }\end{array}$ & Intensity & Prevalence & Abundance \\
\hline \multirow[t]{8}{*}{$\begin{array}{l}\text { Batrachocamallanus slomei } \\
\text { Southwell et Kirshner, } 1937\end{array}$} & Imvubu Lodge, KwaZulu Natal & $\begin{array}{l}28^{\circ} 47^{\prime} 34^{\prime \prime} \mathrm{S} \\
26^{\circ} 03^{\prime} 02^{\prime \prime} \mathrm{E}\end{array}$ & Vegetated pond & 15 & 1 & $7 \%$ & 0.1 \\
\hline & Potchefstroom, North-West & $\begin{array}{l}26^{\circ} 45^{\prime} 20^{\prime \prime} \mathrm{S} \\
27^{\circ} 03^{\prime} 38^{\prime \prime} \mathrm{E}\end{array}$ & Shallow vlei (marshland) & 9 & $14[10](1-37)$ & $100 \%$ & 13.7 \\
\hline & Modimolle, Limpopo & $\begin{array}{l}24^{\circ} 26^{\prime} 18^{\prime \prime} \mathrm{S} \\
28^{\circ} 26^{\prime} 13^{\prime \prime} \mathrm{E}\end{array}$ & Ornamental garden pond & 6 & $18[14](2-45)$ & $83 \%$ & 15.2 \\
\hline & $\begin{array}{l}\text { Jacana Estate, White River, } \\
\text { Mpumalanga }\end{array}$ & $\begin{array}{l}25^{\circ} 20 ' 21 " \mathrm{~S} \\
31^{\circ} 01^{\prime} 21^{\prime \prime} \mathrm{E}\end{array}$ & Dam in vlei (marshland) & 8 & $9[8](4-16)$ & $63 \%$ & 5.6 \\
\hline & $\begin{array}{l}\text { Tasselberry, White River, } \\
\text { Mpumalanga }\end{array}$ & $\begin{array}{l}25^{\circ} 19^{\prime} 55^{\prime \prime} \mathrm{S} \\
31^{\circ} 02^{\prime} 36^{\prime \prime} \mathrm{E}\end{array}$ & Ornamental garden pond & 6 & $24[26](9-37)$ & $100 \%$ & 24.3 \\
\hline & Dullstroom, Mpumalanga & $\begin{array}{l}25^{\circ} 23^{\prime} 53^{\prime \prime S} \\
30^{\circ} 02^{\prime} 17^{\prime \prime} \mathrm{E}\end{array}$ & Dam in mountain stream & 10 & $13[12](1-30)$ & $100 \%$ & 13.0 \\
\hline & Letsitele, Limpopo & $\begin{array}{l}23^{\circ} 47^{\prime} 56 " \mathrm{~S} \\
30^{\circ} 11^{\prime} 42^{\prime \prime} \mathrm{E}\end{array}$ & Vegetated pond & 10 & $6[5](2-12)$ & $100 \%$ & 5.5 \\
\hline & Hermanus, Western Cape & $\begin{array}{l}34^{\circ} 22^{\prime} 13^{\prime \prime S} \\
19^{\circ} 15^{\prime} 25^{\prime \prime} \mathrm{E}\end{array}$ & Vegetated pond & 7 & $3[2](1-6)$ & $57 \%$ & 1.6 \\
\hline \multirow[t]{2}{*}{$\begin{array}{l}\text { Camallanus kaapstaadi } \\
\text { Southwell et Kirshner, } 1937\end{array}$} & Dullstroom, Mpumalanga & $\begin{array}{l}25^{\circ} 23^{\prime} 53^{\prime \prime} \mathrm{S} \\
30^{\circ} 02^{\prime} 17^{\prime \prime} \mathrm{E}\end{array}$ & Dam in mountain stream & 10 & $4[4](1-8)$ & $80 \%$ & 3.4 \\
\hline & $\begin{array}{l}\text { Site A, Cape Town, Western } \\
\text { Cape }\end{array}$ & $\begin{array}{l}33^{\circ} 50^{\prime} 21 " \mathrm{~S} \\
18^{\circ} 36^{\prime} 01^{\prime \prime} \mathrm{E}\end{array}$ & Urban recreational pond & 5 & $2[2](1-2)$ & $60 \%$ & 1.0 \\
\hline $\begin{array}{l}\text { Camallanus xenopodis } \\
\text { Jackson et Tinsley, } 1995\end{array}$ & Dullstroom, Mpumalanga & $\begin{array}{l}25^{\circ} 23^{\prime} 53^{\prime \prime} \mathrm{S} \\
30^{\circ} 02^{\prime} 17^{\prime \prime} \mathrm{E}\end{array}$ & Dam in mountain stream & 10 & $6[6](1-13)$ & $90 \%$ & 5.3 \\
\hline \multirow[t]{2}{*}{ No camallanids present } & $\begin{array}{l}\text { Site B, Cape Town, Western } \\
\text { Cape }\end{array}$ & $\begin{array}{l}33^{\circ} 50^{\prime} 08^{\prime \prime} \mathrm{S} \\
18^{\circ} 33^{\prime} 10^{\prime \prime} \mathrm{E}\end{array}$ & Urban recreational pond & 14 & $\mathrm{n} / \mathrm{a}$ & $\mathrm{n} / \mathrm{a}$ & $\mathrm{n} / \mathrm{a}$ \\
\hline & Rondepan, Limpopo & $\begin{array}{l}23^{\circ} 46^{\prime} 10^{\prime \prime S} \\
29^{\circ} 24^{\prime} 17^{\prime \prime} \mathrm{E}\end{array}$ & Dam in inundated grass & 7 & $\mathrm{n} / \mathrm{a}$ & $\mathrm{n} / \mathrm{a}$ & $\mathrm{n} / \mathrm{a}$ \\
\hline
\end{tabular}

were aligned using the ClustalW tool in Mega (V.7) software and trimmed to the shortest alignment (497 nucleotides of $28 \mathrm{~S}$ and 312 nucleotides of cox 1 genes). The Bayesian analyses were run with the following nucleotide substitution model settings: 1set nst $=6$, rates $=$ invgamma, ncat $=4$, shape $=$ estimate, inferrates $=$ yes and basefreq $=$ empirical, that correspond to a general time reversible model GTR $+\mathrm{G}+\mathrm{I}$. The nucleotide substitution model was determined using JModelTest (V.2.1.7) software. Further analysis was provided using next parameters: $\mathrm{mcmc}$ ngen $=200,000$ for $28 \mathrm{~S}$ alignments and ngen $=100,000$ for $\operatorname{cox} 1$ alignments, samplefreq $=100$, printfreq $=100$ and diagnfreq $=1,000$. Trees were visualised using the FigTree (V. 1.4.3) software.

\section{RESULTS}

Batrachocamallanus slomei Southwell et Kirshner, 1937

Fig. 1

Ho st: Xenopus laevis Daudin (Amphibia: Anura: Pipidae).

L o c a lit i e s : KwaZulu Natal, Mpumalanga, Limpopo, NorthWest, Western Cape (see Table 1).

Site of infection: Stomach, oesophagus.

Representative DNA sequences: MG948463 (cox1), MG947390 (28S).

General description. Body small, usually coiled ventrally, comparatively thick, with maximum width at level of anterior third. Cuticle with prominent transverse striations. Apical (Fig. 1B): oral opening rounded, surrounded by six flat elevations (two larger lateral and four submedian); six labial papillae; four cephalic papillae; two amphids. Buccal capsule with well sclerotised walls, some- what longer than wide with conspicuous basal ring and without internal ridges (Fig. 1A,C). Muscular oesophagus opening surrounded with three tooth-like projections of basal ring (Fig. 1C). Muscular oesophagus club-shaped with elongated posterior bulb. Glandular oesophagus somewhat longer than muscular oesophagus, almost cylindrical but slightly widened posteriorly. Nerve ring encircling muscular oesophagus at level of its proximal third. Excretory pore opening at level of muscular oesophageal mid-length. Minute papilliform deirids situated at level of excretory pore. Intestine straight, narrow. Rectum straight, with thin walls (Fig. 1E). Tail tapering with numerous mucrons in females (Fig. 1E) and rounded tip in males (Fig. 1D).

Male (morphometry based on 20 specimens). Body 1.94-2.80 mm (2.32 mm) long, 96-157 (120) maximum width. Buccal capsule 93-112 (102) long, including basal ring 7-14 (11) long, 53-75 (61) wide with maximum width 67-94 (76) at mid-length and minimum width 26-53 (35) at anterior extremity. Minimum and maximum thickness of buccal capsule walls close to oral opening 2-4 (2.5) and to basal ring 4-10 (6). Muscular oesophagus 188-282 (235) long, 9-12\% (10\%) of body length; 33-56 (43), 33-43 (38) and 60-97 (75) wide at anterior, mid-length and bulb level, respectively. Glandular oesophagus 208-366 (301) long, 10-15\% (13\%) of body length; 46-81 (58), 32-68 (52) and 32-88 (65) wide at anterior, mid-length and posterior level, respectively. Nerve ring at 153-200 (177) from anterior extremity. Excretory pore and deirids situated at 127-280 (217), 6-11\% (9\%) of body length and 213-260 (238), 9-12\% (10\%) of body length from anterior extremity, respectively. Posterior end coiled ventrally, provided 


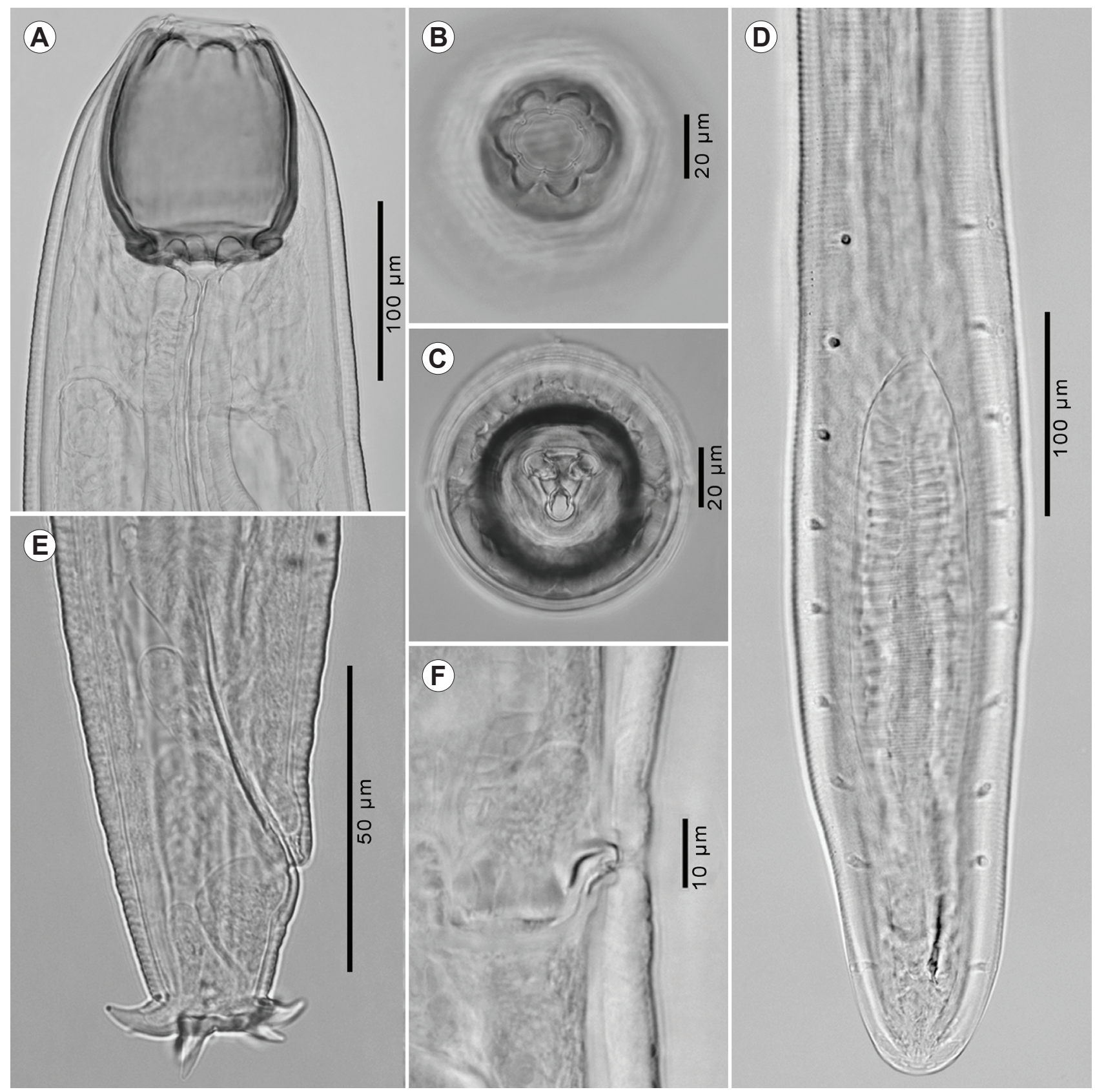

Fig. 1. Batrachocamallanus slomei Southwell et Kirshner, 1937 from Xenopus laevis Daudin, photomicrographs. A - anterior end, female, lateral view; B - anterior end, male, apical view; $\mathbf{C}$ - anterior end, male, optical section at base of buccal capsule level; D - caudal end, male, ventral view; $\mathbf{E}$ - posterior end, female, lateral view; $\mathbf{F}$ - part of female genital system at vulva level, lateral view.

with well-developed vesicular caudal alae supported by pedunculate papillae near mid-length. Posterior part of alae slightly elevated and joined on ventral surface forming pseudosucker. Tail conical with rounded tip, 37-50 (42) long. Caudal region possessing pedunculate papillae (Fig. 1D): eight pairs of precloacal; one pair of adcloacal and three pairs of postcloacal. Three pairs of sessile papillae surrounding cloaca (one slightly anterior to and two somewhat posterior to). Spicules unequal, simple-shaped with sharply pointed distal ends. Right spicule clearly visible, 90-115 (101) long; left one less sclerotised, 15-37 (25) long. Gubernaculum absent.

Female (morphometry based on 30 specimens). Body $1.67-3.51 \mathrm{~mm}(2.87 \mathrm{~mm} \pm 0.54 \mathrm{~mm})$ long, 163-284 (225 \pm 39) maximum width. Buccal capsule 105-138 (122 \pm 9 ) long, including basal ring 10-17 (14 \pm 2$)$ long, 63-90 (76 $\pm 7)$ wide; maximum width $86-113(103 \pm 6)$ at mid-length and minimum width 34-71 (48 \pm 9 ) at anterior extremity. Minimum and maximum thickness of buccal capsule walls

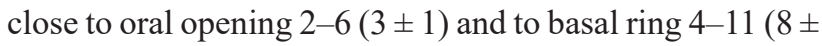
2). Muscular oesophagus $242-337(288 \pm 22)$ long, $8-17 \%$ $(10 \% \pm 2 \%)$ of body length; $38-72(51 \pm 8), 40-60(49 \pm 5)$ and 68-116 (95 \pm 12$)$ wide at anterior, mid-length and bulb level, respectively. Glandular oesophagus 248-526 (369 \pm 62) long, 9-18\% (13\% $\pm 2 \%)$ of body length; 48-78 (65 $\pm 8), 38-76(60 \pm 9)$ and 57-108 $(78 \pm 15)$ wide at anterior, mid-length and posterior level, respectively. Nerve ring at 161-221 (206 \pm 14$)$ from anterior extremity. Excretory 

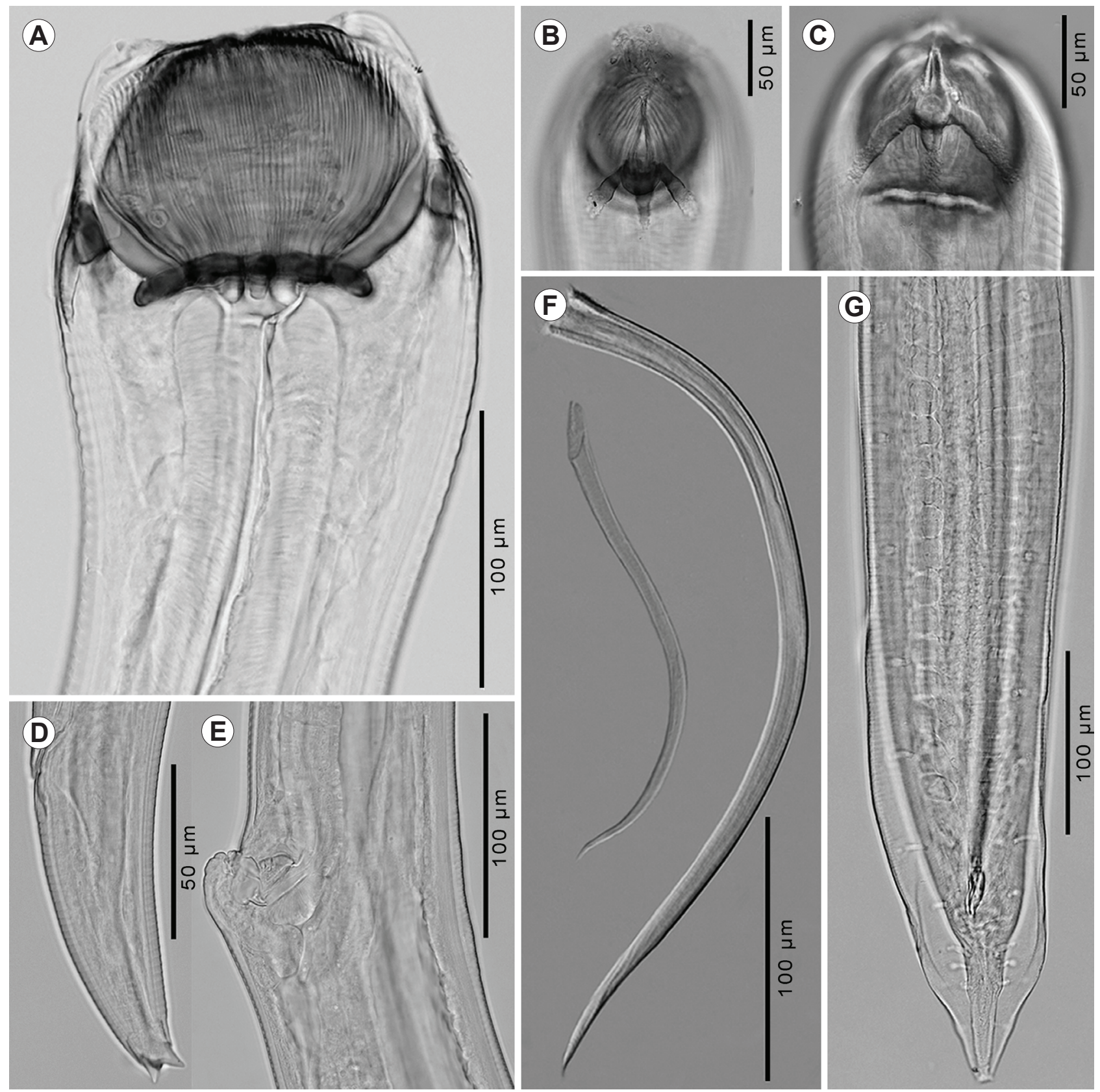

Fig. 2. Camallanus kaapstaadi Southwell et Kirshner, 1937 from Xenopus laevis Daudin, photomicrographs. A - anterior end, male, lateral view; B - dorsal trident, male, lateral view; C - ventral trident, male, lateral view; D - posterior end, female, lateral view; $\mathbf{E}$ - part of female genital system at vulva level, lateral view; $\mathbf{F}$ - left and right spicules, lateral view; $\mathbf{G}$ - caudal end, male, ventral view.

pore and deirids situated at 184-326 (246 \pm 27$), 7-13 \%$, $(9 \% \pm 2 \%)$ of body length, and 196-298 (242 \pm 27$), 6-11 \%$ $(9 \% \pm 1 \%)$ of body length, from anterior extremity, respectively. Vulva (Fig. 1F) situated near body mid-length at $1.13-1.94 \mathrm{~mm}(1.61 \mathrm{~mm} \pm 0.27 \mathrm{~mm})$ from anterior extremity; $42-60 \%(54 \% \pm 3 \%)$ of body length. Tail 34-59 (48 \pm 6) long with five to seven mucrons at its tip (Fig. 1E).

Camallanus kaapstaadi Southwell et Kirshner, 1937

Fig. 2

Ho st: Xenopus laevis Daudin (Amphibia: Anura: Pipidae). L o c a liti es: Mpumalanga, Western Cape (see Table 1). Site of infection: Oesophagus, stomach.

\section{Representative DNA sequences: $\quad$ MG948461 (cox1), MG947391 (28S).}

General description. Medium-sized worms, coiled dorsally, body comparatively thick with maximum width at anterior third level. Cuticle with prominent transverse striations along whole body, except at buccal capsule. Apical: oral opening transversely slit; four conspicuous submedian cephalic papillae. Buccal capsule consisting of two valves each supported with numerous primarily completed ridges (Fig. 2A,B). Four sclerotised plates situated on external surface of valves near their anterior margin. Thick sclerotised basal ring present at buccal capsule base. Buccal capsule valves supported by two prominent tridents on 
dorsal and ventral side (Fig. 2B,C). Each trident consisted of three posteriorly directed prongs. Central prongs somewhat longer than sublateral ones. Tridents usually unequal, beginning at level of buccal capsule mid-length and ending slightly posterior to level of basal ring. Muscular oesophagus club-shaped with elongated posterior bulb. Glandular oesophagus almost equal in length to muscular oesophagus, cylindrical to $3 / 4$ length with slightly widened posterior quarter. Nerve ring encircling muscular oesophagus at level of its anterior quarter. Excretory pore opening on ventral side somewhat posterior to nerve ring level. Deirids minute, papilliform, situated at level of posterior third of muscular oesophagus. Intestine and rectum straight, narrow. Tail tapering.

Male (morphometry based on nine specimens). Body 2.66-3.28 mm (3.04 mm) long, 102-171 (149) wide. Buccal capsule valves 62-100 (83) long, 65-118 (106) wide, supported by 16-31 (26) ridges, of which 7-10 (8) incomplete. Basal ring 7-14 (10) long, 52-89 (77) wide. Dorsal trident 51-72 (66) long and 15-23 (19) wide in lateral projection, ventral one 48-67 (58) long and 17-23 (20) wide.

Muscular oesophagus 261-381 (338.4) long, 9.8-12.3\% (11.1\%) of body length; 53-63 (58.8), 46-61 (53.9) and 58-95 (86.1) wide at anterior, mid-length and bulb level, respectively. Glandular oesophagus 275-461 (368.5) long, 9.1-14.9\% (12.1\%) of body length; 35-96 (61.5), 44-82 (59.5) and 42-76 (59.9) wide at anterior, mid-length and posterior level, respectively. Nerve ring, excretory pore and deirids (distance to deirids measured in three specimens) at 150-246 (182.9), 191-277 (216.1) and 226-257 (241.7) from anterior extremity, respectively.

Caudal alae narrow, ventrolateral, supported by papillae: six pairs of pre-cloacal pedunculated papillae (Fig. $2 \mathrm{G}$ ); one pair of pedunculated papillae at cloaca level; five pairs of post-cloacal pedunculated papillae (three prominent pairs somewhat posterior to cloaca and two pairs of small papillae close to tail end). Additionally, two pairs of sessile ad-cloacal papillae situated slightly anterior and posterior to cloaca. Spicules unequal, simple-shaped with sharpened tips (Fig. 2F). Right spicule prominent, 287-468 (422.5) long; left one less sclerotised (measured in three specimens), 198-220 (211.7) long. Tail conical with two minute spines on tip (Fig. 1G), 74-104 (91.5) long, 2.5$3.2 \%(3.0 \%)$ of body length.

Female (morphometry based on nine specimens). Generally larger than males. Body $2.80-5.13 \mathrm{~mm}$ (3.62 mm) long, 201-293 (242) wide. Buccal capsule valves 95-149 (116) long, 81-174 (147) wide, supported by 31-38 (34) ridges, of which 7-10 (9) incomplete. Basal ring 9-20 (13) long, 71-125 (106) wide. Dorsal trident 72-102 (86) long and 21-29 (25) wide in lateral projection, ventral one 6388 (76) long and 14-35 (24) wide.

Muscular oesophagus 373-451 (414) long, 7-14\% (12\%) of body length; 58-87 (76), 46-79 (69) and 97-140 (123) wide at anterior, mid-length and bulb level, respectively. Glandular oesophagus 313-531 (390) long, 6-15\% (11\%) of body length; 50-91 (72), 49-91 (66) and 52-105 (79) wide at anterior, mid-length and posterior level, respectively. Nerve ring, excretory pore and deirids (distance to deirids measured in five specimens) at 183-242 (220), 229-307 (266) and 261-375 (306) from anterior extremity, respectively.

Vulva post-equatorial, opening posterior to distinct projection of body wall at $1.67-2.64 \mathrm{~mm}(2.16 \mathrm{~mm})$ from anterior extremity (Fig. 2E), 51-63\% (60\%) of body length. Tail tapering 124-179 (143) long, 3-5\% (4\%) of body length, bearing three mucrons 5-11 (8) long at its tip (Fig. 2D).

\section{Camallanus xenopodis Jackson et Tinsley, 1995}

Figs. 3, 4

Host: Xenopus laevis Daudin (Amphibia: Anura: Pipidae).

L oc a lity: Western Cape (see Table 1).

Site of infection: Intestine.

Representative DNA sequences: MG948462 (cox1), MG947389 (28S).

General description. Medium-sized worms, coiled dorsally, body comparatively thick with maximum width at anterior third level. Cuticle with transverse striations clearly visible from level of basal ring to posterior extremity. Apical: oral opening transversely slit; four conspicuous submedian cephalic papillae. Buccal capsule comparatively small, consisting of two valves, supported by longitudinal ridges, with four thin plates on anterior margin, sclerotised basal ring at base and two tridents (Figs. 3B-D, $4 \mathrm{~A}-\mathrm{C}$ ). Tridents prominent, equal in shape, almost equal in size, with central prongs somewhat longer than sublateral ones (Figs. 3C,D, 4B,C). Tridents beginning at level of buccal capsule mid-length, ending somewhat posterior to basal ring. Muscular oesophagus club-shaped with elongated posterior bulb. Glandular oesophagus almost same length as muscular oesophagus, cylindrical along almost entire length with slightly widened or narrowed posterior quarter. Position of nerve ring varying within level of muscular oesophagus anterior third. Excretory pore opening on ventral side somewhat posterior to nerve ring level. Deirids minute, papilliform, situated at level of muscular oesophageal mid-length. Intestine and rectum straight, narrow. Tail tapering.

Male (description based on four specimens). Body 2.88-3.30 mm (3.04 mm) long, 113-127 (118) wide (Fig. 3A). Buccal capsule valves 62-70 (66) long, 72-82 (77) wide, supported by 18-22 (20) ridges, of which 4-6 (5) incomplete. Basal ring 5-11 (9) long, 50-55 (53) wide. Dorsal trident (Figs. 3C, 4B) 57-69 (62) long and 10-18 (15) wide at lateral projection, ventral one (Figs. 3D, 4C) 51-63 (59) long and 9-16 (13) wide.

Muscular oesophagus 257-305 (284) long, 9-11\% (9\%) of body length; 43-51 (45.5), 38-45 (41) and 59-74 (65) wide at anterior, mid-length and bulb level, respectively. Glandular oesophagus 209-313 (271) long, 7-11\% (9\%) of body length; 39-61 (47), 38-58 (49) and 34-73 (53) wide at anterior, mid-length and posterior level, respectively. Nerve ring, excretory pore and deirids (distance to deirids measured in one specimen) at 140-159 (149), 184211 (198) and 213 from anterior extremity, respectively. 

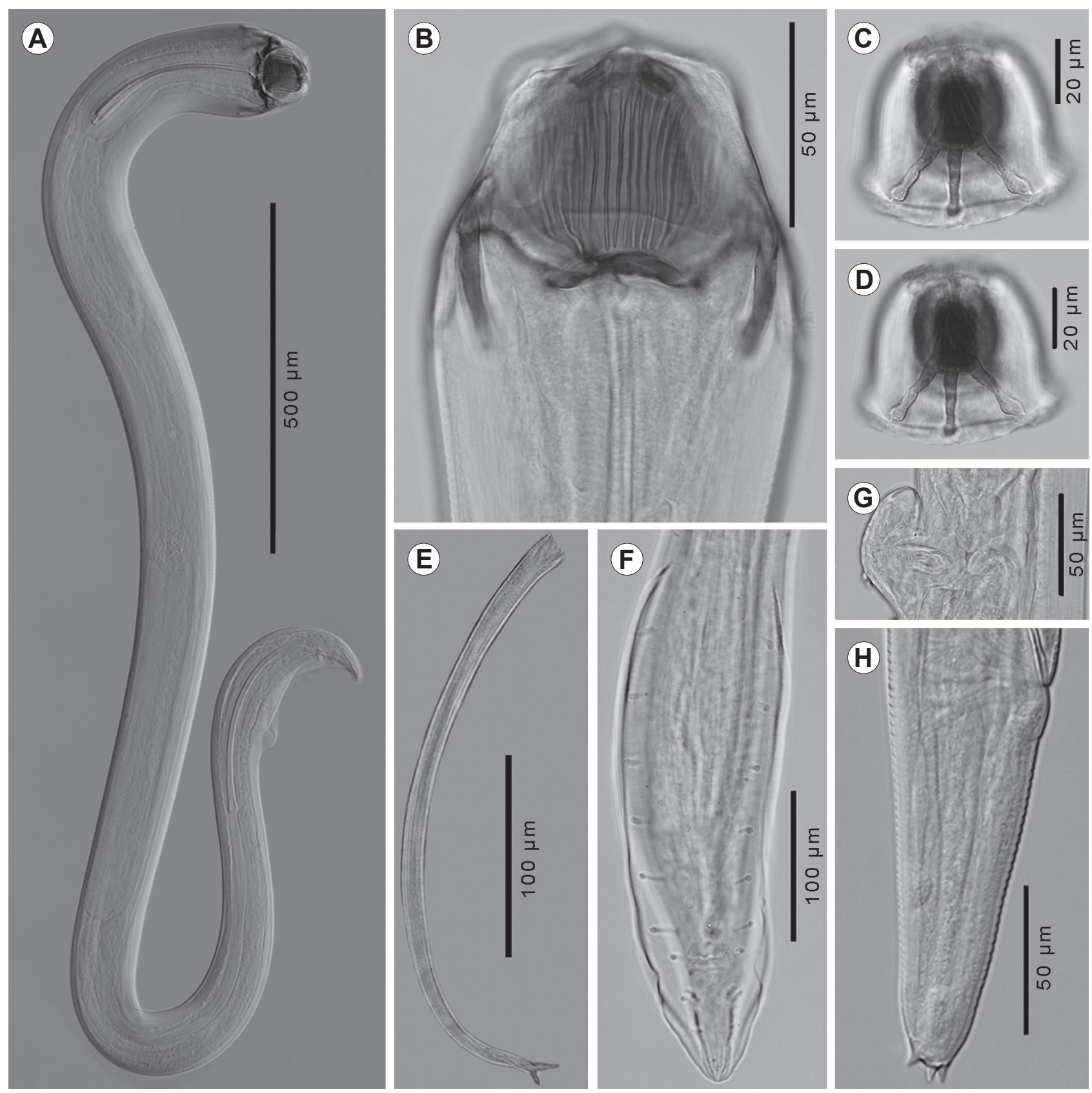

Fig. 3. Camallanus xenopodis Jackson et Tinsley, 1995 from Xenopus laevis Daudin, photomicrographs. A - male, general view; $\mathbf{B}$ - anterior end, female, lateral view; C - dorsal trident, male, lateral view; D - ventral trident, male, lateral view; $\mathbf{E}$ - right spicule, lateral view; F - caudal end, male, ventral view; $\mathbf{G}$ - part of female genital system at vulva level, lateral view; $\mathbf{H}$ - posterior end, female, lateral view.

Caudal alae comparatively narrow, ventrolateral, supported by papillae (Figs. 3F, 4F): six pairs of pre-cloacal pedunculated papillae; one pair of pedunculated papillae at cloaca level; four pairs of post-cloacal pedunculated papillae (two prominent pairs somewhat posterior to cloaca, one pair at tail mid-length and one pair of small papillae close to tail end). Two pairs of sessile adcloacal papillae situated slightly anterior and posterior to cloaca. Spicules unequal. Right spicule (Figs. 3E, 4E) 323-356 (335) long with short conical process directed dorsally at its tip. Left spicule shorter, 96-98 long (measured in two specimens), poorly sclerotised, with simple-shaped sharpened tip (Fig. 4D). Tail tapering with smoothly rounded tip (Figs. 3F, 4F).
Female (description based on 24 specimens). Generally larger than males. Body $3.33-5.13 \mathrm{~mm}(4.20 \mathrm{~mm})$ long, 136-241 (177) wide. Buccal capsule valves 73-85 (81) long, 73-104 (93) wide, supported by 14-22 (18) ridges, of which 3-7 (5) incomplete. Basal ring 8-14 (11) long, 62-74 (67) wide. Dorsal trident 53-93 (76) long and 11-25 (16) wide at lateral projection, ventral one 61-85 (75) long and 12-20 (16) wide.

Muscular oesophagus 326-433 (353) long, 7-12\% (9\%) of body length; 48-68 (58), 39-59 (51) and 76-104 (89) wide at anterior, mid-length and bulb level, respectively. Glandular oesophagus 310-455 (374) long, 7-11\% (9\%) of body length; 42-84 (61), 51-111 (71) and 48-85 (66) 

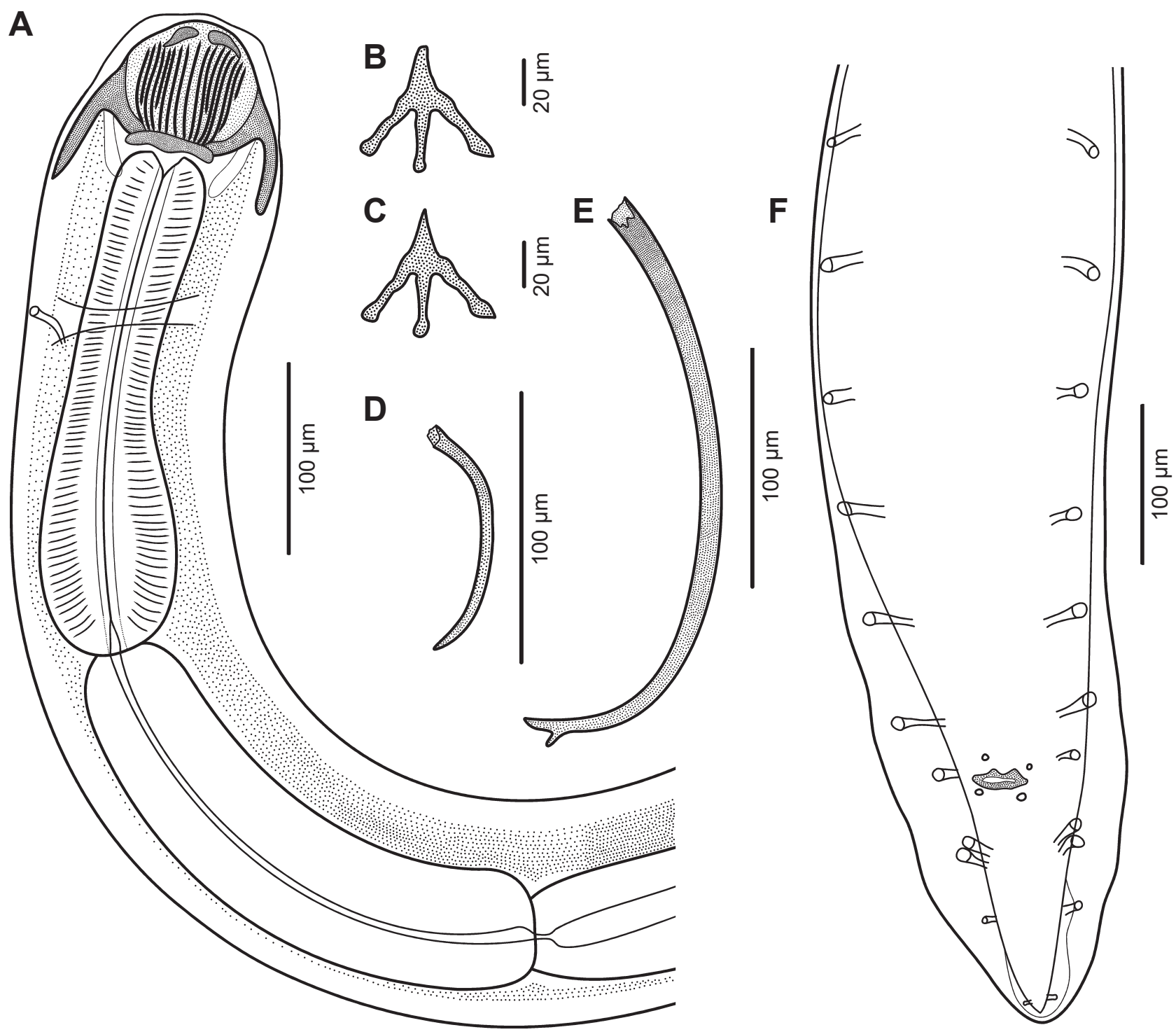

Fig. 4. Camallanus xenopodis Jackson et Tinsley, 1995 from Xenopus laevis Daudin, male, line-drawings. A - anterior end, lateral view; B - dorsal trident, lateral view; C - ventral trident, lateral view; D - left spicule, lateral view; $\mathbf{E}$ - right spicule, lateral view; $\mathbf{F}$ - caudal end, ventral view.

wide at anterior, mid-length and posterior level, respectively. Nerve ring, excretory pore and deirids (distance to deirids measured in eight specimens) at 171-212 (186), 210-256 (228) and 180-291 (247) from anterior extremity, respectively.

Vulva opening posterior to distinct projection of body wall almost at level of mid-body at $1.72-2.59 \mathrm{~mm}$ $(2.14 \mathrm{~mm})$ from anterior extremity (Fig. 3G), 47-54\% (51\%) of body length. Tail tapering 94-165 (129) long, $3-4 \%$ (3\%) of body length, bearing three mucrons 5-7 (6) long at its tip (Fig. 3H).

\section{Molecular analysis}

For all three species, partial 28S gene and coxl fragments were sequenced. Only for five species of Camallanus from Australian freshwater turtles partial sequences of the 28S subunit are available in GenBank. These species were used for the phylogenetic analysis and Serpinema octorugatum (Baylis, 1933) was used as outgroup since it represents the most related genus to Camallanus (see Kuzmin et al. 2011). The resulting tree (Fig. 5) confirmed the interrelationships among C. tuckeri Kuzmin, Tkach, Snyder et Maier, 2009, C. sprenti Kuzmin, Tkach, Snyder et Bell, 2011, C. waelhreow Rigby, Sharma, Hechinger, Platt et Weaver 2008, C. nithoggi Rigby, Sharma, Hechinger, Platt et Weaver 2008 and C. beveridgei Kuzmin, Tkach, Snyder et Bell, 2011 showed by Kuzmin et al. (2011) and showed their distant relations to C. kaapstaadi and C. xenopodis.

The coxl sequences are available for only two species of Camallanus, namely C. cotti Fujita, 1927 and C. hypophthalmichthys Dogel et Akhmerov, 1959, originally described from fishes in the Eastern Palaearctic (Moravec et al. 2004, Moravec and Justine 2006). For this analysis, Spirocamallanus istiblenni Noble, 1966 was used as outgroup. Phylogenetic tree topology showed distant relation- 


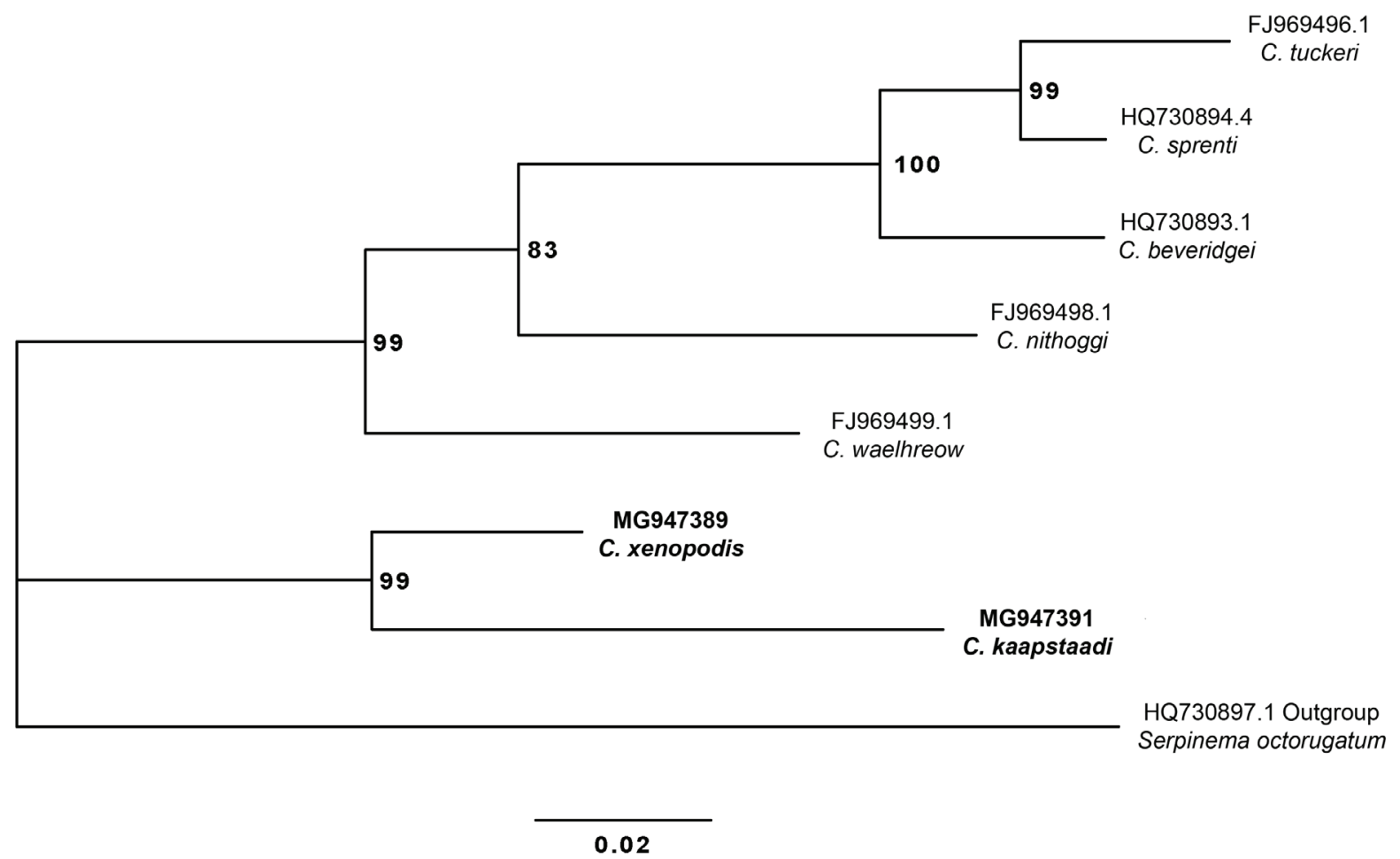

Fig. 5. Phylogenetic tree of seven species of Camallanus Railliet and Henry, 1915 based on partial 28 S sequences.

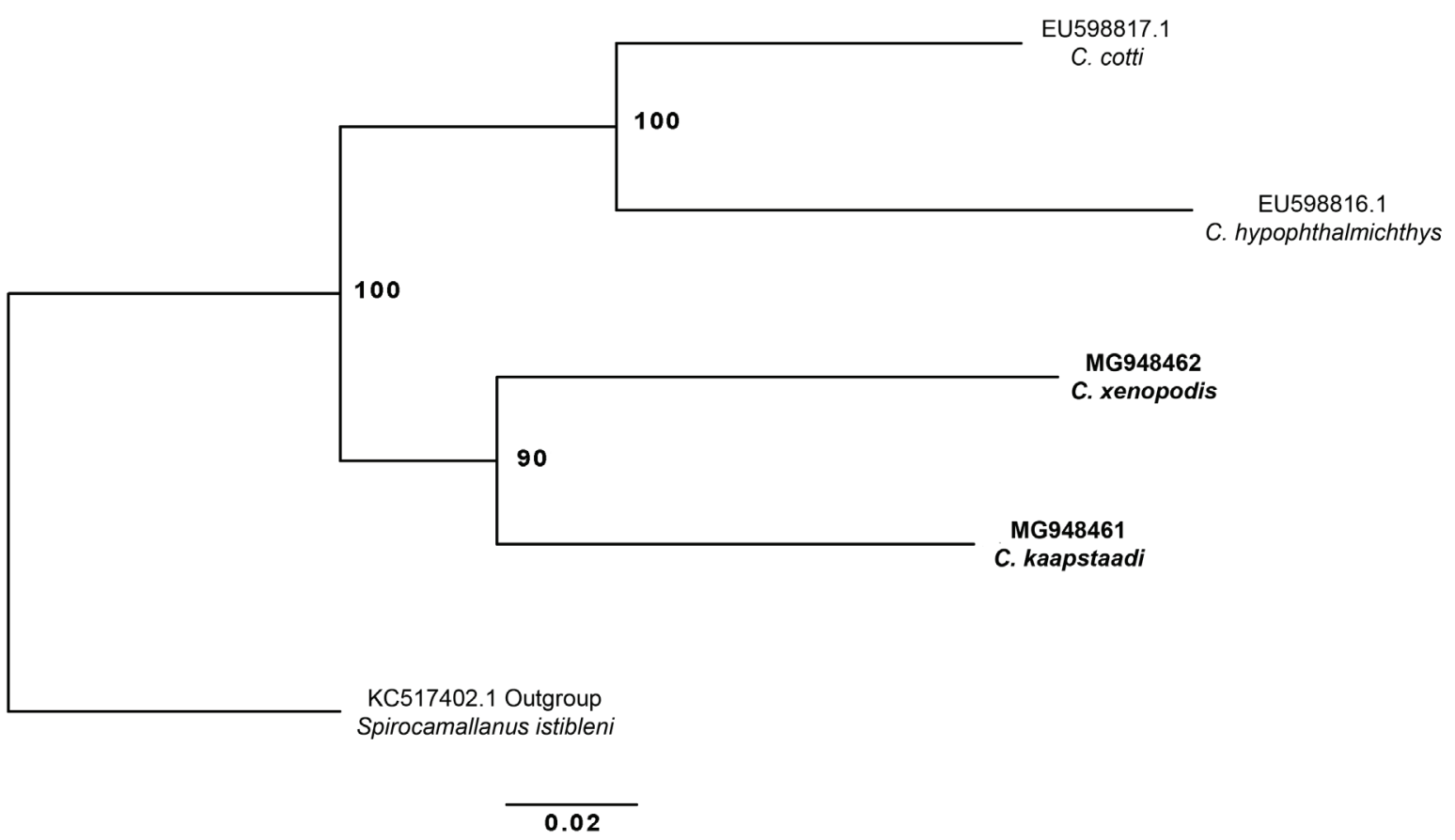

Fig. 6. Phylogenetic tree of four species of Camallanus Railliet and Henry, 1915 based on partial coxl sequences.

ships between Camallanus from African frogs and from Asian fishes which grouped in two strongly supported clades (Fig. 6).

Since neither 28S nor coxl sequences of Batrachocamallanus and Procamallanus are available in GenBank, we are not able to provide phylogenetic analysis for B. slomei. Therefore, representative sequences of that species were simply submitted to GenBank.

\section{DISCUSSION}

During the course of the present study, all three species of camallanid nematodes that have previously been reported from $X$. laevis were found. The most common nematode encountered in the present study was B. slomei, reported from all but three localities with prevalence ranging from $7 \%$ to $100 \%$ (Table 1 ). Conversely, the other two species were found only in two localities. Both C. kaapstaadi and 
C. xenopodis were only found at one locality, namely a series of dams in a mountain stream near Dullstroom (Mpumalanga Province, northern region of South Africa) and only C. kaapstaadi was found at one locality in Cape Town (Western Province). The higher level of nematode diversity at this specific locality where both Camallanus species occur, corresponds with the higher numbers of other parasites (cestodes, monogeneans, digeneans and parasitic arthropods) also found here in comparison to other localities (unpubl. data). In our opinion, this can be explained by the pristine condition of the habitat and lack of human disturbance allowing a better environment for parasite circulation.

Our results confirm the presence of $C$. kaapstaadi in South Africa, in the light of the fact that many previous records are from more northern regions in Africa such as Cameroon, Ghana, Kenya, Nigeria, Rwanda, Sudan, Tanzania, Togo, Uganda and Zimbabwe (Yeh 1960, Thurston 1970, Avery 1971, Tinsley et al. 1979, Jackson and Tinsley 1995a). We found C. xenopodis in the north of South Africa, indicating a less disjunct distribution than the two previous records from the Cape and Kenya suggest (Jackson and Tinsley 1995a). We hypothesise that species of Camallanus in clawed frogs are more widespread than previous studies imply, which will be outlined in further studies.

Batrachocamallanus slomei is found primarily in the stomach, but also in some cases in the oesophagus. Our findings suggest no correlation between the number of nematodes in the stomach and their presence in the oesophagus of the same frog. For example, the frog with the highest infection of $B$. slomei harboured 36 nematodes in the stomach and one nematode in the oesophagus, whereas another frog harboured 15 in the stomach and 17 in the oesophagus. Only one specimen of C. kaapstaadi was found in the stomach while all other specimens inhabited the oesophagus in four frogs sharing this site with B. slomei. Camallanus xenopodis is found only in the intestine of $X$. laevis and no other nematode species are known from this part of the digestive tract.

The reported morphometric characters of $B$. slomei vary widely and correspond to the previously known data (Southwell and Kirshner 1937, Jackson and Tinsley 1995b). Our data slightly expand the existing ranges of body length and width, length of male spicules and distance to vulva from anterior part of body in females. Overall, no differences in morphometric characters of $B$. slomei were observed between localities.

Metrical characters of C. kaapstaadi and C. xenopodis also vary greatly in the studied samples, though most of them fall within the ranges of previously known data (Southwell and Kirshner 1937, Thurston 1970, Jackson and Tinsley 1995a). In the first description of C. kaapstaadi, the authors mentioned that the male left spicule is about $200 \mu \mathrm{m}$ long and less sclerotised than the right one (Southwell and Kirshner 1937). In the later redescription, the authors concurred that the left spicule is poorly visible, but did provide a mean measurement of $155 \mu \mathrm{m}$ (Jackson and Tinsley 1995a). In our specimens, all males of C. kaapstaadi possessed a conspicuous left spicule with a mean length of about $212 \mu \mathrm{m}$. This measurement is longer than that reported for $C$. kaapstaadi from $X$. laevis by Jackson and Tinsley (1995a), probably due to the fact that the specimens in our study were generally bigger ( $2.25 \mathrm{~mm}$ in Jackson and Tinsley (1995a) vs $3.04 \mathrm{~mm}$ in the present study). This discrepancy is also found in the mean measurement of the right spicule $(273 \mu \mathrm{m} v s 423 \mu \mathrm{m})$. At the same time, the left spicule of $C$. xenopodis is truly inconspicuous under light microscopy, even when viewed under high magnification with differential interference contrast (DIC). Nevertheless, the existence of the left spicule is confirmed based on dissection of the caudal part of male.

In our specimens, more females than males of all the species were found, although only in C. xenopodis the females are four times more frequent. Therefore, it is clear why the species was previously described only by females (Jackson and Tinsley 1995a). Males in our samples of C. xenopodis possess several characters, such as structure of buccal capsule, number of ridges on its valves, size and shape of tridents and male right spicule, that are similar to C. mazabukae Kung, 1948. Despite that, two characters, namely number of mucrons (five or at least four in C. mazabukae versus only three in $C$. xenopodis) and the shape of the body ( $C$. mazabukae coiled in the ventral direction whilst all found specimens of $C$. xenopodis coiled dorsally) led us to assign the collected specimens to C. xenopodis.

However, the image of the female tail of C. mazabukae in the original description (Kung 1948) shows that the specimen might really be coiled dorsally. Moreover, the species was described based on only two specimens from the poorly identified host, marked as "bullfrog". Since no Camallanus have been found in the giant bullfrog (Pyxicephalus adsperus Tschudi) or African bullfrog (Pyxicephalus edulis Peters) (Halajian et al. 2013, unpubl. data) before this record or ever since, we suppose that it might represent an opportunistic infection or host misidentification. Given these points, we prefer to identify our specimens as C.xenopodis while the taxonomic status of C. mazabukae should be illuminated by further studies of Camallanus from different amphibians in Africa.

The lack of available DNA alignments in GenBank did not allow us to provide robust phylogenetic analysis of the found species. Both phylogenetic trees (based on partial $28 \mathrm{~S}$ and cox1 alignments) show that species of Camallanus from South African amphibians form a well-supported clade separate from clades of species from Asian fish or Australian freshwater turtles. Unfortunately, we are not able to consider that group as monophyletic, since no data are available of Camallanus from fish (C. polypteri Kabre et Petter, 1997) or turtles (C. chelonius Baker, 1983) in Africa.

Our study revealed only one species of Batrachocamallanus, which limits us in our ability to draw conclusions about the status of the genus. Both the opinions of Jackson and Tinsley (1995b) and Moravec et al. (2006) are based on the morphological characters of nematodes. Recent molecular studies of different groups of nematodes established that morphological characters (especially apomorphies) may appear independently in different lineages 
and are often not suitable for phylogenetic studies (Carreno and Nadler 2003, Tkach et al. 2014). Therefore, in our opinion, the real phylogeny and evolutionary relationships between Camallanidae and their hosts will be illuminated in further molecular studies of more species from different hosts around the globe.
Acknowledgements. The authors wish to express their sincere thanks to Olena Kudlai for her invaluable advice on molecular procedures.

\section{REFERENCES}

Avery R.A. 1971: A preliminary list of parasites collected from reptiles and amphibians in northern Nigeria. Br. J. Herpetol. 4: 217-219.

Carreno R., Nadler S. 2003: Phylogenetic analysis of the Metastrongyloidea (Nematoda: Strongylida) inferred from ribosomal RNA gene sequences. J. Parasitol. 89: 965-973.

Halajian A., Bursey C.R., Goldberg S.R., Luus-Powell W. 2013: Helminths of six species of anurans from the Republic of South Africa: Amietophrynus garmani, Amietophrynus gutturalis, Amietophrynus maculatus, Schismaderma carens (Bufonidae), Amietia angolensis, and Strongylopus grayii (Рyxicephalidae), with a review of South African anuran helminths. Comp. Parasitol. 80: 80-95.

JACKSON J.A., TINSLEy R.C. 1995a: Evolutionary relationships, host range and geographical distribution of Camallanus Railliet \& Henry, 1915 species (Nematoda: Camallaninae) from clawed toads of the genus Xenopus (Anura: Pipidae). Syst. Parasitol. 32: $1-21$.

JACKSON J.A., Tinsley R.C. 1995b: Representatives of Batrachocamallanus n. g. (Nematoda: Procamallaninae) from Xenopus spp. (Anura: Pipidae): geographical distribution, host range and evolutionary relationships. Syst. Parasitol. 31: 159-188.

KunG C.C. 1948: On some new species of spirurids from terrestrial vertebrates, with notes on Habronema mansioni, Physaloptera paradoxa and Hartertia zuluensis. J. Helmintol. 22: 141-161.

Kuperman B.I., Matey V.E., Fisher R.N., Ervin E.L., WarBurton M.L., Bakhireva L., Lehman C.A. 2004: Parasites of the African clawed frog, Xenopus laevis, in southern California, U.S.A. Comp. Parasitol. 71: 229-232.

Kuzmin Y., Tkach V.V., Snyder S.D., Bell J.A. 2011: Camallanus Railliet et Henry, 1915 (Nematoda, Camallanidae) from Australian freshwater turtles with descriptions of two new species and molecular differentiation of known taxa. Acta Parasitol. 56: $213-226$.

Lafferty K.D., Page C.J. 1997: Predation on the endangered tidewater goby, Eucyclogobius newberryi, by the introduced
African clawed frog, Xenopus laevis, with notes on the frog's parasites. Copeia 1997: 589-592.

Moravec F., Justine J.L. 2006: Camallanus cotti (Nematoda: Camallanidae), an introduced parasite of fishes in New Caledonia. Folia Parasitol. 53: 287-296.

Moravec F., Justine J.L., Würzt J., Taraschewski H., Sasal P. 2006: A new species of Procamallanus (Nematoda: Camallanidae) from Pacific eels (Anguilla spp.). J. Parasitol. 92: 130-137.

Moravec F., Nie P., Wang G. 2004: Redescription of Camallanus hypophthalmichthys (Nematoda: Camallanidae) and its first record from fishes in China. J. Parasitol. 90: 1463-1467.

Řehulková E., Baruš V., Gelnar M. 2005: Two remarkable nematodes from the African reedfish Erpetoichthys calabaricus (Polypteriformes: Polypteridae). Helminthologia 42: 149-153.

Southwell T., Kirshner A. 1937: On some parasitic worms found in Xenopus laevis, the South African clawed toad. Ann. Trop. Med. Parasitol. 31: 245-265.

Stromberg P.C., Crites J.L. 1974: Specialization, body volume, and geographical distribution of Camallanidae (Nematoda). Syst. Zool. 23: 189-201.

Thurston, J.P. 1970: Studies on some Protozoa and helminth parasites of Xenopus, the African clawed toad. Rev. Zool. Bot. Africaines. 82: 349-368.

Tinsley R.C. 1996: Parasites of Xenopus. In: R.C. Tinsley and H.R. Kobel (Eds.), The Biology of Xenopus. Clarendon Press, Oxford, pp. 233-261.

Tinsley R.C., Kobel H.R., Fischberg M. 1979: The biology and systematics of a new species of Xenopus (Anura: Pipidae) from the highlands of Central Africa. J. Zool. 188: 69-102.

Tкасн V.V., Kuzmin Y., Snyder S.D. 2014: Molecular insight into systematics, host associations, life cycles and geographic distribution of the nematode family Rhabdiasidae. Int. J. Parasitol. 44: 273-298.

YeH L.S. 1960: On Camallanus johni sp. nov., collected from Xenopus in Tanganyika Territory. J. Helminthol. 34: 103-106.

Cite this article as: Svitin R., Schoeman A.L., du Preez L.H. 2018: New information on morphology and molecular data of camallanid nematodes parasitising Xenopus laevis (Anura: Pipidae) in South Africa. Folia Parasitol. 65: 003. 